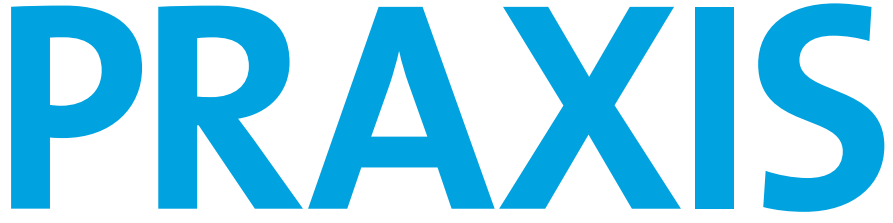

Schweizerische Rundschau für Medizin Revue suisse de la médecine
7. Mai $2014 \cdot 103$. Jahrgang le 7 mai $2014 \cdot 103^{e}$ année

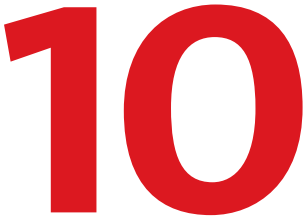

www.praxis.ch ISSN 1661-8157

Therapie der Anaphylaxie

Schwerpunkt: Genomik/Génomique Genomik und personalisierte Medizin

Enjeux éthiques et sociaux de la médecine génomique Patient Privacy in the Genomic Era

Médecine génomique et Maladies infectieuses

Médecine génomique et oncologie

Polyurie-Diagnostik

«Rational Testing» in Zusammenarbeit mit BMJ

PRAXIS ist gelistet in MEDLINE, EMBASE und Scopus

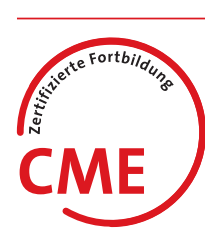

In Zusammenarbeit mit:

BMJ 
Geschäftsführender Herausgeber Prof. Dr. Edouard Battegay, FACP Direktor Klinik und Poliklinik für Innere Medizin

Universitätsspital Zürich

\section{Herausgeber}

Prof. Dr. Johann Steure Horten-Zentrum für praxisorientierte Forschung und Wissenstransfer Universitätsspital Zürich

Prof. Dr. Bernard Waeber Physiopathologie Clinique CHUV Lausanne

Leitende Redaktorin Valérie Herzog

Verlag Hans Huber, Bern

Redaktion

Dr. Barbara Elke

Klinik und Poliklinik für Innere Medizin, Universitätsspital Zürich

Dr. Lorenzo Käser

Medical Education, Forschung

und Lehre, Universitätsspital

Zürich

Dr. Gian Koch

Medizinische Universitätsklinik Kantonsspital Baselland, Liestal

Prof. Dr. Jörg D. Leuppi

Medizinische Universitätsklinik

Kantonsspital Baselland, Liestal

Prof. Dr. Reto Nüesch

Innere Medizin, Spital Schwyz

Dr. Andreas Oestmann

Klinik und Poliklinik

für Allgemeine Innere Medizin

Inselspital Bern

Prof. Dr. Marco Pons

Innere Medizin, Ospedale Civico, Lugano

Prof. Dr. Nicolas Rodondi Medizinische Poliklinik, Klinik für Allgemeine Innere Medizin, Inselspital Bern

Prof. Dr. Thomas Rosemann Institut für Hausarztmedizin, Universitätsspital Zürich

PD Dr. Markus Schneemann Klinik und Poliklinik für Innere Medizin, Universitätsspital Zürich

Prof. Dr. Martin Heinrich Schöni Ambulante Pädiatrie

Kinderklinik, Inselspital Bern

Dr. Hans-Rudolf Schwarzenbach Innere Medizin FMH, Melide

PD Dr. Jan Tuma

Innere Medizin FMH, Uster

\section{Editorial}

551 Sequenzierung des menschlichen Genoms und die Gesellschaft/Décryptage du génome humain et société

Pierre-Yves Maillard

Chef du Département de la santé et de l'action sociale, BAP Lausanne

\section{Continuing Medical Education}

\section{Therapie der Anaphylaxie}

'Martin Meyer, 'Dominik Schaer, ${ }^{2}$ Thomas Harr, 'Florence Vallelian

Klinik und Poliklinik für Innere Medizin, Universitätsspital Zürich';

Unité d'allergologie, Hôpitaux Universitaires de Genève HUG ${ }^{2}$

565 CME-Labor 35: Biochemische Messgrössen des Eisenstoffwechsels

Viola Günther

Institut für Klinische Chemie, Universitätsspital Zürich

605 CME-Rheumatologie 3/Auflösung: Akute Kniegelenksschwellung

Christian Marx, Giorgio Tamborrini

Bethesda-Spital, Basel

607 Primäre Immunthrombozytopenie (ITP)/Antworten

Marc Wehrli, Jeroen S. Goede

Klinik für Hämatologie, Universitätsspital Zürich

\section{Mini-Reviews: Genomik/Génomique}

567 Genomik und personalisierte Medizin

Genomics and Personalized Medicine

Vincent Mooser

Département des Laboratoires, CHUV Lausanne

573 Enjeux éthiques et sociaux de la médecine génomique

Ethical and Social Issues Associated with Genomic Medicine

Gaia Barazzetti, Alain Kaufmann, Lazare Benaroyo

Département universitaire de médecine et santé communautaires, Ethos-

Plateforme interdisciplinaire d'éthique, UNIL-CHUV Lausanne

579 Patient Privacy in the Genomic Era

Datenschutz in der Genomik-Ära

Jean Louis Raisaro, Erman Ayday, Jean-Pierre Hubaux

School of Computer and Communication Sciences, Laboratory for Communications and Applications (LCA1), EPFL Lausanne

587 Médecine génomique et Maladies infectieuses

Genomic Medicine and Infectious Diseases

Jacques Fellay

Faculté des Sciences de la Vie, EPFL, Service des Maladies Infectieuses, CHUV,

Institut Suisse de Bioinformatique, Lausanne 


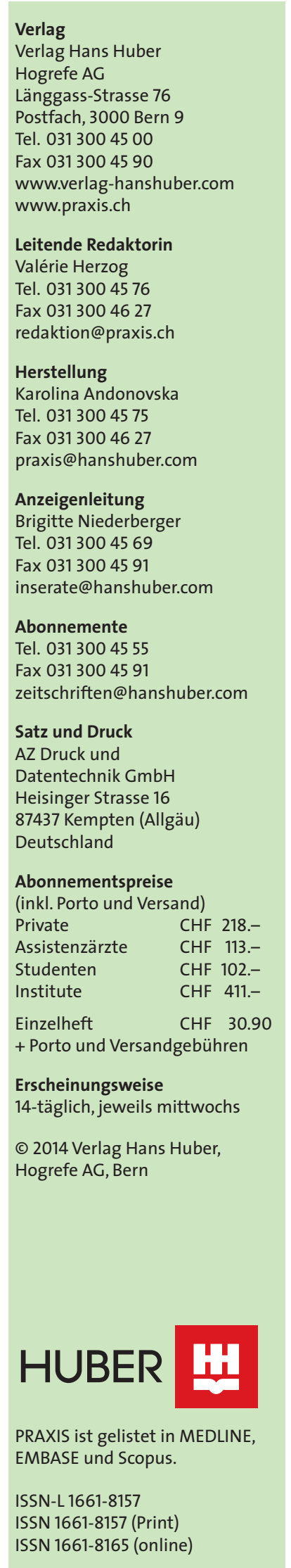

591 Médecine génomique et oncologie

Genomics Medicine and Oncology

Olivier Michielin, George Coukos

Département d'oncologie, CHUV Lausanne

\section{Mini-Review BMJ «Rational Testing»}

\section{Polyurie-Diagnostik}

${ }^{1}$ Adam D. Jakes, ${ }^{2}$ Sunil Bhandari

Leeds Teaching Hospitals NHS Trust, Grossbritannien'; Renal Unit, Hull and

East Yorkshire Hospitals NHS Trust and Hull York Medical School, Grossbritannien ${ }^{2}$

\section{PRAXIS-Journal Club}

\section{Blutungsrisiko von Dabigatran und Vitamin-K-Antagonisten ähnlich} Johann Steurer

Horten-Zentrum für praxisorientierte Forschung und Wissenstransfer, Universitätsspital Zürich

\section{Diät reduziert Reizdarm-Symptome}

Stefan Markun

Horten-Zentrum für praxisorientierte Forschung und Wissenstransfer, Universitätsspital Zürich

\section{Rubriken}

550 Impressum

3. US Vorschau

\section{Magazin}

608 Die Mediziner-Kunstkolumne

609 Mediziner-Literaturrätsel 


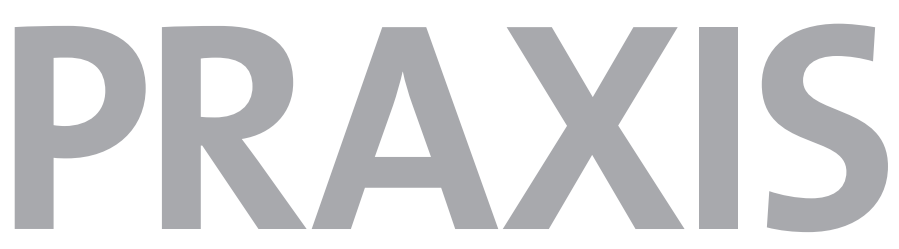

Your article has appeared in a journal published by Hans Huber Publishers.

This e-offprint is provided exclusively for the personal use of the authors.

It may not be posted on a personal or institutional website or to an institutional or disciplinary repository.

If you wish to post the article to your personal or institutional website or to archive it in an institutional or disciplinary repository, please use either a pre-print or a post-print of your manuscript in accordance with the publication release for your article and our "Online Rights for Journal Articles" (http://www.verlag-hanshuber.com/informationen). 
Département universitaire de médecine et santé communautaires, Ethos - Plateforme interdisciplinaire d'éthique, UNIL-CHUV Lausanne

Gaia Barazzetti, Alain Kaufmann, Lazare Benaroyo

\title{
Enjeux éthiques et sociaux de la médecine génomique
}

\author{
Ethical and Social Issues Associated with Genomic Medicine
}

\section{Résumé}

La médecine génomique est souvent présentée comme un nouveau paradigme permettant une prise en charge personnalisée du patient. Englobant à la fois une démarche de recherche et une vision de la médecine du futur, elle pourrait avoir des conséquences importantes sur la manière de diagnostiquer, traiter et prévenir la maladie. Cet article présente quelques grands enjeux éthiques et sociaux soulevés par le développement de la médecine génomique: les implications sur nos conceptions de la maladie et de l'identité, la question de la validité et de l'utilité clinique des analyses du génome, les enjeux liés à la maîtrise de l'information génétique par les soignants et à sa communication aux patients, et la question des coûts pour le système de santé.

Mots-clés: éthique - genetic literacy génomique - médecine personnalisée

\section{Introduction}

Faisant son apparition dans la littérature scientifique au milieu des années 1990, à la suite des premiers succès du Programme Génome Humain, l'expression «médecine génomique» est devenue de plus en plus courante dans la presse et sur le web, souvent comprise comme un synonyme de médecine «personnalisée» ou «individualisée». Alors même qu'il est toujours plus utilisé, ce terme relativement nouveau recouvre des significations multiples et parfois difficiles à cerner.

Selon ses promoteurs, la médecine génomique comprend toute application clinique, actuelle ou potentielle, des techniques de séquençage de nouvelle génération (Next Generation Sequencing - NGS) [1,2]. Les tests génétiques prescrits actuellement n'analysent qu'un seul gène ou des portions d'un seul chromosome à la fois, pour confirmer ou exclure une suspicion de maladie. Le NGS permet par contre d'examiner simultanément un nombre important de gènes, voire l'ensemble de l'ADN codant (Whole Exome Sequencing - WES) et même la totalité du génome (Whole Genome Sequencing - WGS) à la recherche des mutations responsables de maladies génétiques rares ou pouvant contribuer à la survenue de maladies plus communes. Au sens large, le concept de médecine génomique englobe à la fois une démarche de recherche et une vision de la médecine du futur [3]: l'identification des marqueurs génétiques associés à des pathologies rares ou communes permettrait de développer de nouveaux tests diagnostiques, d'envisager de nouveaux traitements, d'améliorer le pronostic et de mettre en place des programmes de prévention.

La médecine génomique est souvent présentée comme un nouveau paradigme permettant une prise en charge personnalisée qui tient compte des spé- cificités génétiques de chaque patient $[1,2]$. Qu'il s'agisse d'une véritable révolution dans les domaines du diagnostic et du traitement, ou plutôt d'une inévitable évolution de la pratique de la génétique médicale $[4,5]$, la médecine personnalisée est à portée de main sur Internet. Depuis quelques années, plusieurs compagnies, dont la plus connue est 23andMe [6], commercialisent sur le Web «directement auprès du consommateur» des tests de prédisposition à une quantité de maladies communes, du diabète aux pathologies cardio-vasculaires [7]. Déjà aujourd'hui, alors que la valeur prédictive de beaucoup de ces tests doit encore être validée, le conseil du médecin pourrait donc être sollicité par des patients ayant acheté en ligne leur screening génomique utilisant la méthode du Genome Wide Association Studies (GWAS) [8].

Nous proposons dans cet article une discussion de quelques grands enjeux éthiques et sociaux soulevés par le développement de la médecine génomique, notamment: les implications sur nos conceptions de la maladie et de l'identité, la question de la validité et de l'utilité clinique des analyses du génome, les enjeux liés à la maîtrise de l'information génétique par les soignants et à sa communication aux patients, et la question des coûts de la médecine génomique.

Les abréviations utilisées dans l'article:

NGS Next Generation Sequencing

WES Whole Exome Sequencing

WGS Whole Genome Sequencing 


\section{Conceptions de la maladie et de l'identité}

Les progrès réalisés dans la recherche en génomique ont des implications importantes sur notre manière de caractériser et de traiter la maladie et sur notre représentation de l'identité biologique.

La génomique est en train de reconfigurer la nosologie, qui sera de moins en moins fondée sur des symptômes ou l'analyse histologique des tissus, et reposera davantage sur les bases moléculaires des pathologies [2]. L'identification des marqueurs génomiques associés aux maladies contribue à en enrichir la nosographie, avec pour conséquence une démultiplication en sous-types de la même pathologie [3]. Ces changements sont déjà avérés en oncologie, où ils commencent à avoir des impacts sur la manière de diagnostiquer et de traiter la maladie [1,2]. Des diagnostics toujours plus précoces des risques génétiques, jusqu'au stade asymptomatique des maladies, permettent d'intervenir en amont des processus pathologiques et dans certains cas de les prévenir. Des marqueurs génomiques commencent à être utilisés dans le cadre de thérapies plus individualisées, permettant de garantir l'efficacité du traitement, de minimiser les effets secondaires et d'optimiser le dosage des médicaments, particulièrement dans certaines chimiothérapies, dans le traitement du sida ou de l'hépatite C [2].

En ce qui concerne notre identité biologique, elle tend à devenir de plus en plus contextuelle, en intégrant non seulement le patrimoine génétique hérité de nos parents, mais aussi les interactions potentielles ou avérées entre ce dernier et les génomes des agents pathogènes que nous abritons et des innombrables populations de microbes qui peuplent notre système digestif, ou microbiome [1].

Sur le plan sociologique, on assiste à l'émergence de nombreuses associations de patients qui sont engagées dans la production simultanée d'une nouvelle identité qui est tout à la fois biologique et sociale. Cette dernière, qui s'incarne notamment au travers du Téléthon, peut donc être appelée «biosociale» dans la mesure ou elle associe étroitement des caractéristiques biologiques spécifiques revendiquées par ces groupes, et des demandes particulières en terme de recherche scientifique, de diagnostic, de thérapeutique, de prise en charge d'un handicap et de politiques sociales $[9,10]$. Qu'il s'agisse de groupes de patients atteints par une forme spécifique de myopathie ou d'une maladie neurodégénérative comme la Chorée de Huntington, ils établissent des liens entre leurs caractéristiques génétiques et la construction sociale de leur identité.

\section{Validité et utilité d'une médecine génomique}

L'intégration de la médecine génomique dans la pratique médicale soulève la question fondamentale de sa pertinence au plan du jugement clinique [4]. En effet, si les progrès de la génomique pourront assurément apporter beaucoup plus de précision concernant les critères permettant l'élaboration d'un jugement clinique circonstancié, ils ne peuvent constituer, comme tout progrès technologique, les seuls fondements du jugement clinique. S'il est vrai que la pratique médicale contemporaine comporte une part de plus en plus importante d'applications techniques, à laquelle les progrès de la génomique contribuent, elle ne saurait toutefois se réduire à cela. Comme de nombreux travaux de philosophie de la médecine l'ont mis en évidence tout au long du $20^{\text {ème }}$ siècle, la pratique médicale ne peut être considérée comme la simple application d'éléments cognitifs issus des sciences cliniques [11]. La pratique se différencie du savoir scientifique par le fait qu'elle implique toujours une prise de décision responsable qui opère un choix éthique entre diverses options thérapeutiques [12].

Pour pouvoir apprécier les bénéfices attendus de la médecine génomique et pour qu'elle puisse apporter une contribution à la pratique médicale, les nouveaux tests génétiques devraient satisfaire à des critères de "validité» et d'«utilité» clinique $[2,13,14]$. La validité clinique d'un test réside dans sa valeur prédictive, c'est-à-dire dans le degré de certitude de la corrélation entre un résultat positif et la survenue d'une maladie. A l'heure actuelle, l'une des difficultés majeures dans l'évaluation de la validité clinique du séquençage concerne l'interprétation des résultats, surtout en ce qui concerne le diagnostic des maladies génétiques multifactorielles [15]. En effet, l'interprétation des tests peut varier considérablement dans le temps, au fur et à mesure que les connaissances en génomique progressent. Le médecin devrait disposer des connaissances nécessaires pour évaluer la pertinence des résultats pour le suivi médical du patient et devrait faire régulièrement appel à des cliniciens spécialisés en médecine génétique pour vérifier et mettre à jour l'interprétation des données génomiques [15]. Les organisations professionnelles et les instances politiques seraient également concernées, en tant que responsables de la formation des praticiens, du développement de recommandations et de la mise à disposition de ressources documentaires adaptées pour l'évaluation et la révision périodique de la valeur prédictive des tests.

En ce qui concerne l'utilité clinique de la médecine génomique, les chercheurs et cliniciens sont de plus en plus conscients que les bénéfices attendus pour les patients ne devraient pas simplement être mesurés sous l'angle de la réduction de la morbidité et de la mortalité générales, mais aussi en termes d'amélioration du bien-être individuel [1,2]. L'évaluation de l'utilité clinique des tests génomiques inclut donc une appréciation de l'«utilité personnelle», pour le patient, de l'information génomique dans son suivi médical $[5,14,16]$. En effet, surtout dans le cas de maladies multifactorielles, l'information concernant un risque génétique ne conduit pas forcément le patient à chan- 
ger ses habitudes et à améliorer son hygiène de vie [17]. La pratique du conseil génétique a déjà montré que la relation entre connaissance des risques et bienêtre individuel est moins évidente qu'on pourrait le croire. Ainsi, l'intégration des données génomiques dans la prévention, le diagnostic et le traitement des maladies nécessite une meilleure compréhension des modalités selon lesquelles les patients et les soignants s'approprient et font usage de ces informations [1].

\section{Communication de l'information génomique et "genetic literacy"}

Par rapport aux tests génétiques classiques, la particularité des nouvelles techniques de séquençage du génome est de générer systématiquement une très grande quantité d'informations, concernant non seulement les facteurs de risque génétique visés, mais aussi de l'information excédentaire qui n'était pas recherchée, qu'on appelle résultats «inattendus» (incidental findings), «non sollicités» ou «secondaires». En cas de résultats ayant une pertinence clinique incertaine ou inconnue, la communication de l'information génomique au patient soulève plusieurs questions éthiques, sociologiques, psychologiques et juridiques $[18,19]$. Si le principe du respect de l'autonomie exigerait que l'ensemble des résultats soit communiqué, le principe de bienfaisance appelle à plus de prudence car l'incertitude des résultats peut susciter des préoccupations injustifiées chez le patient. Question d'autant plus aigue que les risques génétiques peuvent concerner les apparentés. La variabilité de l'interprétation des résultats dans le temps, en fonction du progrès des connaissances, pose des questions concernant le devoir éventuel des professionnels de recontacter le patient dans le futur.

Afin de limiter la quantité d'éventuels résultats secondaires et faciliter la communication de l'information géno- mique, la Société Européenne de Génétique Humaine suggère de privilégier des analyses ciblées, par panels de gènes associés à une utilité clinique avérée [20]. Les recommandations européennes prévoient également que toute analyse génomique dans le contexte clinique soit précédée par une évaluation des bénéfices et des risques liés à la communication des résultats, et par un conseil génétique. L'importance d'impliquer les patients dans la décision sur le retour des résultats est d'ailleurs confirmée par des études récentes qui montrent l'intérêt des individus à avoir accès à l'information qui les concernent et à participer à l'appréciation de sa valeur [21].

Face aux progrès rapides de la génomique, il devient de plus en plus urgent que les professionnels de la santé, les médecins de premier recours en particulier, acquièrent les connaissances nécessaires (genetic literacy) à évaluer la pertinence des analyses génomiques pour la prise en charge des patients. Les praticiens devraient pouvoir disposer d'outils adaptés, sous forme de guidelines et de ressources d'information pour l'évaluation de la validité et de l'utilité clinique des tests. Les patients devraient également être mieux informés sur les implications de ces analyses pour leur santé et mis en garde vis-à-vis des tests commercialisés sur Internet sans aucun suivi médical. Les cliniciens spécialisés en génétique médicale et les conseillers en génétique pourraient jouer un rôle fondamental, notamment dans la sensibilisation des patients et des praticiens aux enjeux liés à l'interprétation et à la communication des résultats.

\section{Coûts de la médecine génomique et allocation des ressources}

Le progrès des technologies de séquençage a permis de générer des données génomiques à des coûts toujours plus bas. Selon une estimation du National Human Genome Research Institute
(NHGRI) aux Etats-Unis, le coût du séquençage est passé de 100 millions de \$ en 2001 à moins de 10000 \$ en 2013 [22], et ces coûts continuent de chuter. Pourtant, la génération des données n'est qu'un élément entrant dans le calcul des coûts réels des analyses génomiques [23]. Pour les mesurer il faut aussi considérer les coûts liés au développement et au management des plateformes de séquençage, au stockage des données, et au travail d'analyse et d'interprétation de ces dernières (downstream analyses); ce sans parler des coûts engendrés par la prise en charge du patient pour lui communiquer les résultats, qui nécessiterait davantage de temps en raison de la quantité et de la complexité des informations à restituer.

La question des coûts du développement d'une médecine génomique personnalisée soulève par ailleurs des interrogations concernant l'allocation des ressources [3]. Les investissements et les efforts consacrés à la médecine génomique devraient être justifiés par des preuves solides de son utilité clinique et ne devraient pas priver de ressources précieuses d'autres secteurs de la pratique médicale. Par contre, si ces coûts devaient dépasser les ressources à disposition dans le secteur public, le risque serait grand que les progrès soient réalisés essentiellement dans le secteur privé, avec pour conséquence probable l'essor d'un marché de tests et de traitements accessibles seulement aux patients les plus aisés.

\section{Conclusions}

La promesse d'une médecine «personnalisée» par le biais des acquis de la génomique ne doit pas nous faire oublier que la personnalisation dont il est question ici relève avant tout d'une capacité accrue à identifier les caractéristiques biologiques individuelles. Ces développements pourront contribuer à l'élaboration d'un jugement clinique circonstancié relevant d'un soin personnalisé, qui constitue le noyau éthique 


\section{Messages-clés}

- La médecine génomique devrait satisfaire à des critères de «validité» et d'«utilité» clinique pour qu'elle puisse apporter des réels bénéfices à la pratique médicale.

- Face aux progrès rapides de la génomique, il est essentiel que les professionnels de la santé acquièrent les connaissances nécessaires (genetic literacy) à évaluer la pertinence des analyses génomiques pour la prise en charge des patients.

- L'évaluation des coûts réels de la médecine génomique est nécessaire pour apprécier sa contribution potentielle à la pratique médicale.

- La gestion des enjeux éthiques et sociaux soulevés par le transfert de la génomique dans la clinique nécessite une démarche interdisciplinaire, associant sciences biomédicales et sciences humaines, et qui devrait de plus impliquer les patients et les citoyens.

\section{Key messages}

- Die medizinische Genomik muss Kriterien der klinischen Validität sowie der klinischen Nützlichkeit genügen, damit sie für die praktische Medizin einen realen Nutzen hervorbringen kann.

- Angesichts der rasanten Fortschritte der Genomik ist es für alle Gesundheitsspezialisten von grosser Wichtigkeit, sich die notwendigen Kenntnisse (genetic literacy) anzueignen, um die Relevanz von Genom-Analysen für die Behandlung des Patienten einschätzen zu können.

- Die Evaluierung der tatsächlichen Kosten der genomischen Medizin ist wichtig, um ihren potenziellen Beitrag an die praktische Medizin einschätzen zu können.

- Für die Bewältigung ethischer und sozialer Herausforderungen durch den Einzug der Genomik in die Klinik braucht es ein interdisziplinäres Vorgehen unter Einbezug der biomedizinischen und humanmedizinischen Wissenschaften wie auch der Patienten und Bürger.

d'une pratique médicale dès son origine hippocratique. En ce sens la médecine génomique constitue davantage une évolution qu'une révolution [4].

Comme toute approche technologique et clinique émergente, la médecine génomique nous confronte à de nombreuses incertitudes qui soulèvent des enjeux éthiques et sociaux. Il est donc essentiel de disposer de dispositifs de réflexion et de dialogue qui ne réduisent pas a priori le spectre des problèmes possibles et qui intègrent les transformations en cours. La gestion de ces incertitudes et le transfert de la génomique dans la clinique ne peuvent se faire sans la contribution de l'ensemble des professions et insti- tutions concernées. Il s'agit donc d'une démarche éminemment interdisciplinaire, associant sciences biomédicales et sciences humaines (sociologie, philosophie, psychologie, économie, anthropologie, droit), et qui devrait de plus impliquer les futurs usagers d'une médecine génomique en voie de constitution: les patients et les citoyens. Plusieurs centres de recherche engagés dans le développement de biobanques génomiques, en particulier au Canada, au Royaume-Uni et aux Etats-Unis [24-26], ont d'ailleurs mis en place des dispositifs de gouvernance participative associant des patients à la définition des usages pertinents de ces outils, par exemple pour ce qui concerne la définition des priorités de recherche ou les modalités de retour des résultats aux malades.

\section{Remerciements}

Nous remercions Florence Fellmann et Francesco Panese pour leurs propositions constructives.

\section{Zusammenfassung}

Die medizinische Genomik wird oft als neues Paradigma dargestellt, das eine personalisierte Therapie des Patienten erlaubt. Sie umfasst gleichzeitig eine Forschungsart und eine Zukunftsvision der Medizin und könnte einen gewaltigen Einfluss auf Diagnose, Therapie und Prävention haben. Dieser Artikel beleuchtet wichtige ethische und soziale Fragen, die durch die Genom-basierte Medizin aufgeworfen werden: Die Auswirkungen auf unser Verständnis der Krankheiten, die Frage der Gültigkeit und des klinischen Nutzens der Analyse des Genoms, die Kontrolle der genetischen Information von den Betreuenden und ihre Kommunikation zum Patienten sowie die Frage der Kosten für das Gesundheitssystems.

Schlüsselwörter: Ethik - genetic literacy - Genomik - personalisierte Medizin

\section{Abstract}

Genomic medicine is often presented as a new paradigm for personalized healthcare. Encompassing both a translational approach in research and a vision of future medical practice, genomic medicine may have important impact on the way healthcare professionals diagnostics, treat and prevent diseases. We discuss some ethical and social issues raised by the prospect of genome-based medical practice, namely: changing definitions of disease and 
identity, assessment of clinical validity and utility of genome screening, mastery of genomic information by healthcare professionals and its communication to patients, and questions related to the costs of genomic medicine for future healthcare.

Key words: ethics - genetic literacy genomics - personalised medicine

\section{Adresses de correspondance}

Gaia Barazzetti, PhD

Unité d'éthique

Département universitaire de médecine et santé communautaires, UNIL-CHUV

Ethos - Plateforme interdisciplinaire

d'éthique, UNIL

1015 Lausanne

Gaia.Barazzetti@unil.ch

\section{Bibliographie}

1. Green ED, Guyer MS: Cherting a course for genomic medicine from base pairs to bedside. Nature 2011; 470: 204-213.

2. McCarthy JJ, McLeod HL, Ginsburg GS: Genomic medicine: a decade of success, challenges, and opportunities. Science Trans Med 2013; 5: 189sr4.

3. Boenink M:Tensions and opportunities in convergence: shifting concepts of disease in emerging molecular medicine. Nanoethics 2009; 3: 243-255.

4. Steele FR: Personalized medicine: something old, something new. Personalized Medicine 2009; 6: 1-5.

5. Offit K: Personalized medicine: new genomics, old lessons. Hum Genet 2011; 130: $3-14$.

6. https://www.23andme.com; consulté le 20.01.2014
7. Dvoskin R, Kaufman D: Tables of direct-to-consumer genetic testing companies and conditions tested - August 2011. Genetics and Public Policy Center, Washington, DC, 2011. http:// www.dnapolicy.org/pub.reports.php?action=detail\&report_id $=28$

8. McGuire AL, Burke W: An unwelcome side effect of direct-to-consumer personal genome testing. Raiding the medical commons. JAMA 2008; 300: 2669-2671.

9. Kaufmann A. In: Joye C. (Dir.). Handicap et diagnostic à l'aube de la vie: espoir ou dérive? Regards croisés sur le diagnostic préimplantatoire: Le rôle des associations de patients dans la définition de l'identité sociale et biologique des personnes. Genève; Editions Médecine \& Hygiène: 2012, 79-87.

10. Rabinow P. In: Essays on the Anthropology of reason: Artificiality and enlightenment: from sociobiology to biosociality. Princeton; Princeton University Press: 1996. 91-111.

11. Benaroyo L: Peut-on accepter les progrès en sciences biomédicales sans progrès en éthique? J Int Bioethique 2013; 24: 23-42.

12. Benaroyo L: Ethique et responsabilité en médecine. Genève; Médecine \& Hygiène: 2006.

13. Foster MW, Mulvihill JJ, Sharp RR: Evaluating the utility of personal genomic information. Genet Med 2009; 11: 570-574.

14. Edelman E, Eng C: A practical guide to interpretation and clinical application of personal genomic screening. BMJ 2009; 339: b4253.

15. Shirts BH, Parker LS: Changing interpretations, stable genes: responsibilities of patients, professionals, and policy makers in the clinical interpretation of complex genetic information. Genet Med 2008; 10: 778-783.

16. Evaluation of Genomic Applications in Practice and Prevention (EGAPP) Working Group: The EGAPP initiative: lessons learned. Genetics In Medicine 2013; advance online publication 8 August 2013. doi:10.1038/gim.2013.110

17. Henrikson NB, Bowen D, Burke W: Does genomic risk information motivate people to change their behavior? Genome Med 2009; 1: 37 .

18. Clarke A: What do we mean by "genetic testing" in 2013? Bioethica Forum 2013; 6: 105-106.

19. Clarke A, Thirlaway K: Genetic couselling for personalized medicine. Hum Genet 2011;130: 27-31.

20. Van El CG, Cornel MC, Borry P, et al.: Whole-genome sequencing in health care. Recommendations of the European Society of Human Genetics. Eur J Hum Genet 2013; 21: 580-584.

21. Townsend A, Adam S, Birch PH, Lohn Z, Rousseau F, Friedman JM: "I want to know what's in pandora's box": comparing stakeholder perspectives on incidental findings in clinical whole genomic sequencing. Am J Med Genet Part A 2012; 158A: 2519-2525.

22. http://www.genome.gov/sequencingcosts/; consulté le 20.01.2014.

23. Sboner A, Xinmeng JM, Greenbaum D, Auerbach R, Gerstein $M$ : The real cost of sequencing: higher than you think! Genome Biol 2011; 12: 125.

24. Avard D, Bucci LM, Burgess MM, Kaye J, Heeney C: Public health genomics (PHG) and public participation: points to consider. Journal of Public Deliberation 2009; 5: Article 7. Available at: http://www.publicdeliberation.net/jpd/vol5/iss1/art7

25. O'Doherty KC, Hawkins AK, Burgess MM: Involving citizens in the ethics of biobank research: informing institutional policy through structured public deliberation. Soc Sci Med 2012; 75: 1604-1611.

26. Olson JE, Ryu E, Johnson KJ, et al: the Mayo Clinic biobank: a building block for individualized medicine. Mayo Clin Proc 2013; 88: 952-962. 